OPEN ACCESS

Edited by:

Youssef Zeidan,

American University of Beirut,

Lebanon

Reviewed by:

Stephan Bodis,

Kantonsspital Aarau, Switzerland John M. Watkins,

University of lowa, United States

*Correspondence:

Steven H. Lin

shlin@mdanderson.org

Specialty section: This article was submitted to

Radiation Oncology,

a section of the journal

Frontiers in Oncology

Received: 14 June 2017 Accepted: 12 September 2017 Published: 27 September 2017

Citation:

Verma V, Allen PK and Lin SH (2017) Evaluating Factors for Prophylactic

Feeding Tube Placement in

Gastroesophageal Cancer Patients Undergoing Chemoradiotherapy.

Front. Oncol. 7:235

doi: 10.3389/fonc. 2017.00235

\section{Evaluating Factors for Prophylactic Feeding Tube Placement in Gastroesophageal Cancer Patients Undergoing Chemoradiotherapy}

\author{
Vivek Verma', Pamela K. Allen ${ }^{2}$ and Steven H. Lin ${ }^{2 *}$ \\ ${ }^{1}$ Department of Radiation Oncology, University of Nebraska Medical Center, Omaha, NE, United States, \\ ${ }^{2}$ Department of Radiation Oncology, University of Texas M.D. Anderson Cancer Center, Houston, TX, United States
}

Purpose: Though better studied in head/neck cancers, there are currently no studies on timing of feeding tube (FT) placement in patients with gastroesophageal cancer. This study sought to discern characteristics of patients who used versus did not use a prophylactic $\mathrm{FT}(\mathrm{pFT})$, and also analyzed factors associated with placement of FTs during chemoradiotherapy (CRT).

Methods/materials: From 1998 to 2013, 1,329 patients underwent neoadjuvant CRT, of which 323 received an FT. Patients for whom FTs were placed prior to treatment due to tumor occlusion or substantial weight loss $(n=130)$, and those with FTs placed following treatment $(n=43)$ were excluded. One hundred patients had pFTs placed, and 50 underwent placement during CRT. The following was collected for each patient: demographic/ patient information, oncologic/treatment characteristics, and CRT tolerance.

Results: No significant differences were found in any parameter between cohorts that used $(n=66)$ versus did not use a pFT $(n=34)$; on univariate and multivariate analyses, no pretreatment characteristic associated with using a pFT. When compared with patients who used a pFT ( $n=66)$, those who required an FT during CRT $(n=50)$ had lower body mass index $(p=0.045)$, underwent higher-dose radiotherapy $(p=0.003)$, and received induction chemotherapy $(p=0.031)$. On multivariate analysis, receipt of induction chemotherapy and greater weight loss and esophagitis during treatment were associated with placement of FTs during CRT $(p<0.05)$.

Conclusion: Of our cohort who received pFTs, there were no clinical factors that predicted for their use. Patients must be closely monitored for weight loss and esophagitis when receiving CRT in order to intervene prior to further worsening of toxicities.

Keywords: esophageal cancer, gastric tube, nutrition, chemotherapy, radiation therapy

\section{INTRODUCTION}

Feeding tubes (FTs) can be used for nutritional support in cancer patients and are either placed prophylactically or as a response to toxicities during or after oncologic therapy. Though most commonly used in head and neck cancers, timing of use-especially prophylactically-is controversial and without consensus at present (1-3). In comparison, FTs are inserted much less frequently for 
gastroesophageal (GE) neoplasms. Incidences have been reported as high as $63 \%(4)$, but also as low as $8 \%(5)$; however, absolute numbers remain low. Nevertheless, poor nutritional status can affect subsequent treatment options and outcomes including those of surgery (6).

No evidence has been published to date examining factors associated with FT insertion at various time points, as well as evaluating prophylactic placement of FTs in GE cancers. As such, the National Comprehensive Cancer Network insinuates that FT placement be considered on a case-by-case basis, especially in conditions of low caloric intake and/or esophageal obstruction (7).

In this study, we sought to examine the population of GE cancers that received FTs, treated at a high-volume, tertiary care academic medical center. Specifically, our study had two principal goals. First, because prophylactic FTs (pFTs) may or may not be utilized, we aimed to ascertain factors associated with needing to use a pFT. Second, a comparison of factors was made between patients who used a pFT and those who required an FT during radiotherapy (RT), in efforts to delineate a high-risk population that could benefit from aggressive supportive care and possibly a lower threshold for FT placement during RT.

\section{MATERIALS AND METHODS}

\section{Patient Population}

This single-institutional analysis examined 1,329 patients with cancer of the esophagus or GE junction treated with concurrent chemoradiotherapy (CRT) as part of neoadjuvant therapy (1998-2013). Of these patients, the analyzed population consisted of those who underwent FT placement, which numbered 323 patients. Each patient provided informed consent to place an FT if recommended. Figure 1 displays patient subgroups. Exclusion criteria for this study included those patients who underwent FT insertion after RT $(n=43)$; though many of these were from CRTrelated toxicities, FTs in many others were placed in anticipation

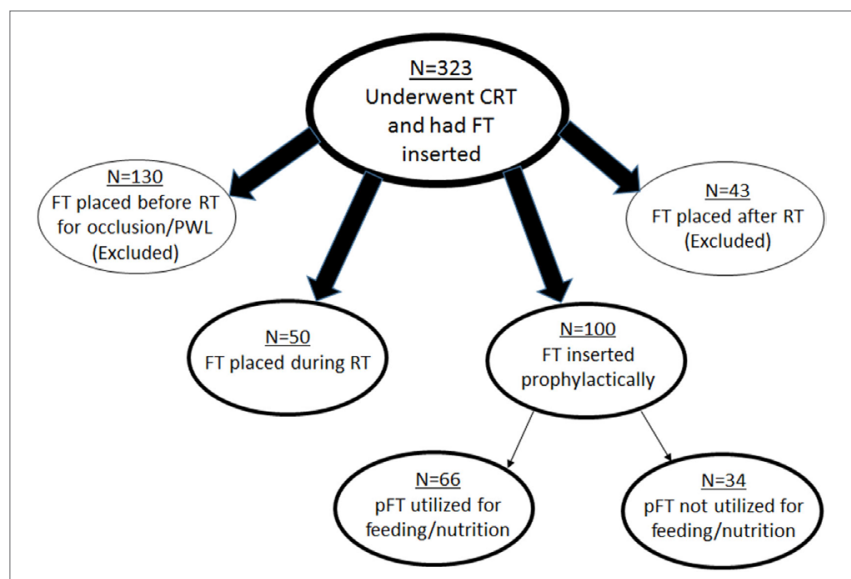

FIGURE 1 | Schematic representation of patient subgroups in this study. CRT, chemoradiotherapy; FT, feeding tube; RT, radiotherapy; PWL, profound weight loss; pFT, prophylactic feeding tube. of surgery and not directly as a result of toxicities. Similarly, 130 patients had FT placed prior to RT specifically owing to partial or complete luminal occlusion from the malignancy and/or with profound weight loss (PWL) (greater than 10\% weight loss in past 3-6 months). Because this constituted a heterogeneous patient population for the purposes of our analysis, they were additionally excluded.

These criteria left 150 patients for further investigation. Of these patients, 50 had placement during RT, and 100 were placed prophylactically. Reasons for FT placement during RT included odynophagia, dysphagia, decreased appetite, and nausea/vomiting. pFT placement was performed at the clinician's discretion, based on clinical concern but not due to any of the aforementioned rationale for FT placement. Whether FTs were utilized for nutrition (defined as at least one administration of enteral feeding) was then recorded; 66 patients used the FT and 34 did not.

Two comparisons between patient subgroups were made in this study: (1) factors associated with use of a pFT $(n=66)$ versus lack thereof $(n=34)$ and (2) characteristics between patients who used a pFT $(n=66)$ versus those who had FT insertion during RT $(n=50)$. For each patient in each subgroup, many parameters were collected, broadly including patient/demographic information, tumor and treatment characteristics, and intra-CRT tolerance.

\section{Oncologic Therapy}

Radiotherapy was administered in three possible modalities: three-dimensional conformal RT, intensity-modulated RT, or proton beam RT. Briefly, for each modality, patients were immobilized using custom molds and simulated in the supine position with four-dimensional computed tomography simulation. Positron emission tomography fusion aided in target volume definition. In general, doses consisted of 41.4-50.4 Gy and were delivered with concurrent chemotherapy, which consisted of various regimens over time and at the medical oncologist's discretion. Patients were seen weekly by the medical and radiation oncologists on treatment, with reassessment of toxicities and potential intervention at each visit. Surgery was performed several weeks after cessation of CRT and most commonly was transthoracic esophagectomy.

\section{Statistical Analysis}

Data analysis was performed using Stata/MP14 statistical software (College Station, TX, USA). The Wilcoxon rank-sum and Fisher's exact tests were used to compare the factors associated with use and lack of use of a pFT, as well as to compare the characteristics of patients who used a pFT against those whom required an FT placed during treatment. Univariate and multivariate logistic regression were performed in order to examine variables associated with placement of FTs in various circumstances. $p$-values $<0.05$ were considered to be statistically significant. Statistical tests were based on a two-sided significance level.

\section{RESULTS}

Table 1 displays characteristics of patients who did $(n=66)$ versus did not utilize $(n=34)$ their pFT. There were no statistically 
TABLE 1 | Clinical characteristics of patients who used versus did not use a pFT.

\begin{tabular}{|c|c|c|c|c|}
\hline Parameter & All patients with pFT $(n=100)$ & Did not use pFT $(n=34)$ & Used pFT $(n=66)$ & $p$-values \\
\hline Median age at diagnosis (years) (range) & $64(21-84)$ & $65(29-84)$ & $63.5(21-84)$ & 0.951 \\
\hline \multicolumn{5}{|l|}{ Gender } \\
\hline Male & $80(80 \%)$ & $29(85 \%)$ & $51(77 \%)$ & \multirow[t]{2}{*}{0.434} \\
\hline Female & $20(20 \%)$ & $5(15 \%)$ & $15(23 \%)$ & \\
\hline Median body mass index (kg/m²) (range) & $26.8(16.5-43.0)$ & $27.3(19.9-35.5)$ & $25.5(16.5-43.0)$ & 0.334 \\
\hline \multicolumn{5}{|l|}{ Karnofsky performance status } \\
\hline 60 & $2(2 \%)$ & $1(3 \%)$ & $1(2 \%)$ & \multirow[t]{5}{*}{0.569} \\
\hline 70 & $6(6 \%)$ & $1(3 \%)$ & 5 (8\%) & \\
\hline 80 & $44(44 \%)$ & $16(47 \%)$ & $28(42 \%)$ & \\
\hline 90 & $40(40 \%)$ & $15(44 \%)$ & 25 (38\%) & \\
\hline 100 & $8(8 \%)$ & $1(3 \%)$ & $7(11 \%)$ & \\
\hline \multicolumn{5}{|l|}{ Location } \\
\hline Upper & $17(17 \%)$ & $8(24 \%)$ & $9(14 \%)$ & \multirow[t]{3}{*}{0.171} \\
\hline Middle & 7 (7\%) & $4(12 \%)$ & $3(5 \%)$ & \\
\hline Lower & $76(76 \%)$ & $22(65 \%)$ & $54(82 \%)$ & \\
\hline Median tumor length (cm) (range) & $6(1-15)$ & $7(2-13)$ & $5(1-15)$ & 0.065 \\
\hline \multicolumn{5}{|l|}{ AJCC clinical T stage } \\
\hline $\mathrm{T} 1$ & $1(1 \%)$ & $0(0 \%)$ & $1(2 \%)$ & \multirow[t]{4}{*}{0.651} \\
\hline $\mathrm{T} 2$ & $9(9 \%)$ & $4(12 \%)$ & $5(8 \%)$ & \\
\hline T3 & $83(83 \%)$ & $29(85 \%)$ & $54(82 \%)$ & \\
\hline $\mathrm{T} 4$ & 7 (7\%) & $1(3 \%)$ & $6(9 \%)$ & \\
\hline \multicolumn{5}{|l|}{ AJCC clinical $N$ stage } \\
\hline NO & 32 (32\%) & $10(29 \%)$ & $22(33 \%)$ & \multirow[t]{2}{*}{0.822} \\
\hline $\mathrm{N} 1$ & $68(68 \%)$ & $24(71 \%)$ & $44(67 \%)$ & \\
\hline \multicolumn{5}{|l|}{ Receipt of induction chemotherapy } \\
\hline No & $47(47 \%)$ & $17(50 \%)$ & $30(45 \%)$ & \multirow[t]{2}{*}{0.679} \\
\hline Yes & $53(53 \%)$ & $17(50 \%)$ & $36(54 \%)$ & \\
\hline \multicolumn{5}{|l|}{ Dysphagia symptoms at diagnosis } \\
\hline No & $28(28 \%)$ & 7 (21\%) & $21(32 \%)$ & \multirow[t]{2}{*}{0.347} \\
\hline Yes & $72(72 \%)$ & 27 (79\%) & $45(68 \%)$ & \\
\hline \multicolumn{5}{|l|}{ Odynophagia symptoms at diagnosis } \\
\hline No & $81(81 \%)$ & $28(82 \%)$ & 53 (80\%) & \multirow[t]{2}{*}{0.999} \\
\hline Yes & $19(19 \%)$ & $6(18 \%)$ & $13(20 \%)$ & \\
\hline Median weight loss at diagnosis (kg) (range) & $3.0(0-27)$ & $1.5(0-20)$ & $3(0-27)$ & 0.238 \\
\hline Median total RT dose (Gy) (range) & $50.4(16.2-66)$ & $50.4(16.2-66)$ & $50.4(32.4-62.8)$ & 0.098 \\
\hline Median PTV volume (cm³) (range) & $800(45-3,080)$ & $798(210-2,168)$ & $801(45-3,080)$ & 0.743 \\
\hline \multicolumn{5}{|l|}{ RT modality } \\
\hline 3DCRT & $47(47 \%)$ & $16(47 \%)$ & $31(47 \%)$ & \multirow[t]{3}{*}{0.835} \\
\hline IMRT & $47(47 \%)$ & $17(50 \%)$ & $30(45 \%)$ & \\
\hline PBT & $6(6 \%)$ & $1(3 \%)$ & $5(8 \%)$ & \\
\hline Median weight loss during RT (kg) (range) & $3.9(0-16.5)$ & $3.0(0-15.0)$ & $4.5(0-16.5)$ & 0.143 \\
\hline
\end{tabular}

pFT, prophylactic feeding tube; AJCC, American Joint Commission on Cancer; PTV, planning target volume; RT, radiotherapy; 3DCRT, three-dimensional conformal radiotherapy; IMRT, intensity-modulated radiotherapy; PBT, proton beam therapy.

significant differences between groups in any parameter analyzed, including performance status, body mass index (BMI) at diagnosis, tumor length/stage, symptoms at diagnosis, target volume size, or RT dose. Univariate analysis, performed in order to examine variables associated with use of a pFT, did not show correlation with any examined variable (Table 2). On multivariate analysis, the only factor associated with use of a pFT was weight loss during treatment [continuous variable; odds ratio (OR) 1.16, $95 \%$ confidence interval (CI) $1.01-1.32, p=0.03$ ]. Hence, no pretreatment parameter was found to be reliably associated with use of a pFT.

Next, attention was shifted to compare patients who used a pFT $(n=66)$ versus those who had an FT placed during CRT $(n=50)$. Results of this comparison are shown in Table 3. Of note, lower BMI (median 25.5 versus 23.9, $p=0.045$ ) and higherdose RT (median 50.4 versus $50.4, p=0.003$ ) corresponded to
FT placement during CRT. This cohort that underwent FT insertion during CRT experienced greater weight loss on treatment (mean 7.6 versus $4.9 \%, p=0.002$ ) and higher toxicity due to both dysphagia $(p<0.001)$ and esophagitis $(p<0.001)$. These patients were also more likely to have missed RT treatments, as three patients missed seven or more sessions. Table 4 displays results of univariate analysis of parameters associated with placement of an FT during RT: induction chemotherapy, RT dose, size of the planning target volume, and toxicities during RT (weight loss, dysphagia, esophagitis). Multivariate analysis revealed that patients undergoing induction chemotherapy were more likely to undergo FT placement during RT (OR 2.46, 95\% CI 1.09-5.57, $p=0.031)$. Other factors included patients suffering greater weight loss during the treatment course (OR 0.31, 95\% CI $0.11-0.90, p=0.031$ ) as well as esophagitis (OR 0.20, 95\% CI $0.06-0.61, p=0.005)$. 
TABLE 2 | Univariate analysis of factors associated with use of a prophylactic feeding tube.

\begin{tabular}{|c|c|c|c|}
\hline Parameter & Odds ratio & $95 \% \mathrm{Cl}$ & $p$-values \\
\hline \multicolumn{4}{|l|}{ Median age at diagnosis } \\
\hline Continuous variable & 1 & $0.97-1.04$ & 0.876 \\
\hline \multicolumn{4}{|l|}{ Gender } \\
\hline Male versus female & 0.59 & $0.19-1.78$ & 0.346 \\
\hline \multicolumn{4}{|l|}{ Body mass index } \\
\hline Continuous variable & 0.98 & $0.89-1.07$ & 0.602 \\
\hline \multicolumn{4}{|c|}{ Karnofsky performance status } \\
\hline Continuous variable & 1.01 & $0.96-1.06$ & 0.668 \\
\hline \multicolumn{4}{|l|}{ Location } \\
\hline Middle versus upper & 0.67 & $0.11-3.93$ & 0.654 \\
\hline Lower versus upper & 2.18 & $0.75-6.38$ & 0.154 \\
\hline \multicolumn{4}{|l|}{ Tumor length } \\
\hline Continuous variable & 0.86 & $0.72-1.01$ & 0.072 \\
\hline \multicolumn{4}{|l|}{ AJCC clinical T stage } \\
\hline T3/4 versus $\mathrm{T} 1 / 2$ & 1.33 & $0.35-5.09$ & 0.674 \\
\hline \multicolumn{4}{|l|}{ AJCC clinical N stage } \\
\hline N1 versus N0 & 0.83 & $0.34-2.05$ & 0.691 \\
\hline \multicolumn{4}{|c|}{ Receipt of induction chemotherapy } \\
\hline Yes versus no & 1.2 & $0.52-2.75$ & 0.666 \\
\hline \multicolumn{4}{|c|}{ Dysphagia symptoms at diagnosis } \\
\hline Yes versus no & 0.56 & $0.21-1.48$ & 0.24 \\
\hline \multicolumn{4}{|c|}{ Odynophagia symptoms at diagnosis } \\
\hline Yes versus no & 1.14 & $0.39-3.34$ & 0.805 \\
\hline \multicolumn{4}{|l|}{ Weight loss at diagnosis } \\
\hline Continuous variable & 1.05 & $0.98-1.14$ & 0.178 \\
\hline \multicolumn{4}{|l|}{ Total RT dose } \\
\hline Continuous variable & 0.96 & $0.90-1.02$ & 0.199 \\
\hline \multicolumn{4}{|l|}{ PTV volume } \\
\hline Continuous variable & 1 & $0.99-1.01$ & 0.613 \\
\hline \multicolumn{4}{|l|}{ RT modality } \\
\hline IMRT versus 3DCRT & 0.91 & $0.39-2.13$ & 0.829 \\
\hline PBT versus 3DCRT & 2.58 & $0.28-24.00$ & 0.405 \\
\hline \multicolumn{4}{|l|}{ Weight loss during RT } \\
\hline Continuous variable & 1.1 & $0.98-1.23$ & 0.117 \\
\hline
\end{tabular}

Cl, confidence interval; AJCC, American Joint Commission on Cancer; RT, radiotherapy; PTV, planning target volume; IMRT, intensity-modulated radiotherapy; 3DCRT, three-dimensional conformal radiotherapy; PBT, proton beam therapy.

\section{DISCUSSION}

Optimal timing of FT placement in GE cancers is controversial from limited evidence. In our cohort of patients, there were no pretreatment predictors that associated with utilization of a pFT. Hence, because none of the analyzed pretreatment patient, oncologic, or treatment characteristics predicted for utilization of a pFT, these data do not argue in favor of pFT placement. Moreover, receipt of more aggressive therapy (e.g., induction chemotherapy) along with greater symptoms (e.g., weight loss, esophagitis) associated with insertion of FT during RT; hence, select patients must be aggressively monitored for symptoms in order to perform early interventions. Not doing so may risk greater symptomatic suffering and potentially even compromised outcomes from missing RT sessions.

In high-risk patients, both patient-reported and physicianappraised symptoms should be continually re-assessed weekly (at minimum) during treatment, although this may not necessarily mean a lower threshold for FT insertion (other measures such as oral supplements may be considered first). This is important
TABLE 3 | Clinical characteristics of patients who used a pFT versus those who received one during $R T$.

\begin{tabular}{|c|c|c|c|}
\hline Parameter & $\begin{array}{l}\text { Used pFT } \\
(n=66)\end{array}$ & $\begin{array}{l}\text { FT during RT } \\
\qquad(n=50)\end{array}$ & $p$-values \\
\hline $\begin{array}{l}\text { Median age at diagnosis (years) } \\
\text { (range) }\end{array}$ & $63.5(21-84)$ & $63.5(22-91)$ & 0.863 \\
\hline Gender & & & 0.999 \\
\hline Male & $51(77 \%)$ & 39 (78\%) & \\
\hline Female & 15 (23\%) & $11(22 \%)$ & \\
\hline $\begin{array}{l}\text { Median body mass index } \\
\left(\mathrm{kg} / \mathrm{m}^{2}\right) \text { (range) }\end{array}$ & $25.5(16.5-43.0)$ & $23.9(17.4-39.6)$ & 0.045 \\
\hline \multicolumn{4}{|l|}{ Karnofsky performance status } \\
\hline 60 & $1(2 \%)$ & $0(0 \%)$ & 0.104 \\
\hline 70 & $5(8 \%)$ & $6(12 \%)$ & \\
\hline 80 & $28(42 \%)$ & $23(46 \%)$ & \\
\hline 90 & $25(38 \%)$ & $21(42 \%)$ & \\
\hline 100 & $7(11 \%)$ & $0(0 \%)$ & \\
\hline \multicolumn{4}{|l|}{ Location } \\
\hline Upper & $9(14 \%)$ & 5 (10\%) & 0.43 \\
\hline Middle & $3(5 \%)$ & $5(10 \%)$ & \\
\hline Lower & $54(82 \%)$ & $40(80 \%)$ & \\
\hline Median tumor length (cm) (range) & $5(1-15)$ & $5(1-10)$ & 0.351 \\
\hline \multicolumn{4}{|l|}{ AJCC clinical T stage } \\
\hline $\mathrm{T} 1$ & $1(2 \%)$ & $1(2 \%)$ & 0.861 \\
\hline T2 & $5(8 \%)$ & $6(12 \%)$ & \\
\hline T3 & $54(82 \%)$ & $38(76 \%)$ & \\
\hline T4 & $6(9 \%)$ & $4(8 \%)$ & \\
\hline TX & $0(0 \%)$ & $1(2 \%)$ & \\
\hline \multicolumn{4}{|l|}{ AJCC clinical N stage } \\
\hline NO & 22 (33\%) & $13(26 \%)$ & 0.54 \\
\hline N1 & $44(67 \%)$ & $36(72 \%)$ & \\
\hline $\mathrm{NX}$ & $0(0 \%)$ & $1(2 \%)$ & \\
\hline \multicolumn{4}{|l|}{$\begin{array}{l}\text { Receipt of induction } \\
\text { chemotherapy }\end{array}$} \\
\hline No & $30(45 \%)$ & 32 (64\%) & 0.061 \\
\hline Yes & $36(54 \%)$ & $18(36 \%)$ & \\
\hline \multicolumn{4}{|l|}{$\begin{array}{l}\text { Dysphagia symptoms at } \\
\text { diagnosis }\end{array}$} \\
\hline No & $21(32 \%)$ & $11(22 \%)$ & 0.296 \\
\hline Yes & $45(68 \%)$ & $39(78 \%)$ & \\
\hline \multicolumn{4}{|l|}{$\begin{array}{l}\text { Odynophagia symptoms at } \\
\text { diagnosis }\end{array}$} \\
\hline No & $53(80 \%)$ & $43(86 \%)$ & 0.467 \\
\hline Yes & $13(20 \%)$ & 7 (14\%) & \\
\hline $\begin{array}{l}\text { Median weight loss at diagnosis } \\
(\mathrm{kg}) \text { (range) }\end{array}$ & $3.0(0-27)$ & $6.8(0-27)$ & 0.074 \\
\hline Median total RT dose (Gy) (range) & $50.4(32.4-62.8)$ & $50.4(16.2-66)$ & 0.003 \\
\hline Median PTV volume (cm³) (range) & $801(45-3,080)$ & $683(118-1,525)$ & 0.106 \\
\hline \multicolumn{4}{|l|}{ RT modality } \\
\hline 3DCRT & $31(47 \%)$ & $19(38 \%)$ & 0.535 \\
\hline IMRT & $30(45 \%)$ & $25(50 \%)$ & \\
\hline PBT & $5(8 \%)$ & $6(12 \%)$ & \\
\hline $\begin{array}{l}\text { Median weight loss during RT } \\
\text { (kg) (range) }\end{array}$ & $4.5(0-16.5)$ & $7.4(0-18.1)$ & 0.002 \\
\hline \multicolumn{4}{|l|}{ Grade of weight loss } \\
\hline 0 & 33 (50\%) & $9(18 \%)$ & 0.001 \\
\hline 1 & $25(37 \%)$ & 27 (54\%) & \\
\hline 2 & $7(11 \%)$ & $12(24 \%)$ & \\
\hline 3 & $0(0 \%)$ & $2(4 \%)$ & \\
\hline Unknown & $1(2 \%)$ & $0(0 \%)$ & \\
\hline \multicolumn{4}{|l|}{ Grade of dysphagia } \\
\hline 0 & $18(27 \%)$ & 7 (14\%) & $<0.001$ \\
\hline 1 & $14(21 \%)$ & $3(6 \%)$ & \\
\hline 2 & $21(32 \%)$ & $11(22 \%)$ & \\
\hline 3 & $13(20 \%)$ & $29(58 \%)$ & \\
\hline
\end{tabular}


TABLE 3 | Continued

\begin{tabular}{lrrr}
\hline Parameter & $\begin{array}{c}\text { Used pFT } \\
(\boldsymbol{n}=\mathbf{6 6})\end{array}$ & $\begin{array}{c}\text { FT during RT } \\
(\boldsymbol{n}=\mathbf{5 0 )})\end{array}$ & $\boldsymbol{p}$-values \\
\hline $\begin{array}{c}\text { Grade of esophagitis } \\
0\end{array}$ & $14(21 \%)$ & $2(4 \%)$ & $<\mathbf{0 . 0 0 1}$ \\
1 & $7(11 \%)$ & $3(6 \%)$ & \\
2 & $33(50 \%)$ & $9(18 \%)$ & \\
3 & $12(18 \%)$ & $36(72 \%)$ & \\
\hline
\end{tabular}

Statistically significant $p$-values are in bold.

pFT, prophylactic feeding tube; RT, radiotherapy; AJCC, American Joint Commission on Cancer; PTV, planning target volume; 3DCRT, three-dimensional conformal radiotherapy; IMRT, intensity-modulated radiotherapy; PBT, proton beam therapy.

TABLE 4 | Univariate analysis of factors associated with placement of a feeding tube during $\mathrm{RT}$ treatments.

\begin{tabular}{|c|c|c|c|}
\hline Parameter & Odds ratio & $95 \% \mathrm{Cl}$ & $p$-values \\
\hline \multicolumn{4}{|l|}{ Median age at diagnosis } \\
\hline Continuous variable & 0.99 & $0.96-1.02$ & 0.631 \\
\hline \multicolumn{4}{|l|}{ Gender } \\
\hline Male versus female & 0.96 & $0.40-2.32$ & 0.926 \\
\hline \multicolumn{4}{|l|}{ Body mass index } \\
\hline Continuous variable & 1.08 & $1.00-1.18$ & 0.064 \\
\hline \multicolumn{4}{|c|}{ Karnofsky performance status } \\
\hline Continuous variable & 1.03 & $0.98-1.08$ & 0.207 \\
\hline \multicolumn{4}{|l|}{ Location } \\
\hline Middle versus upper & 0.33 & $0.06-2.02$ & 0.232 \\
\hline Lower versus upper & 0.75 & $0.23-2.41$ & 0.629 \\
\hline \multicolumn{4}{|l|}{ Tumor length } \\
\hline Continuous variable & 1.13 & $0.96-1.34$ & 0.152 \\
\hline \multicolumn{4}{|l|}{ AJCC clinical T stage } \\
\hline T3/4 versus T1/2 & 1.67 & $0.52-5.31$ & 0.388 \\
\hline \multicolumn{4}{|l|}{ AJCC clinical N stage } \\
\hline N1 versus N0 & 0.72 & $0.32-1.63$ & 0.434 \\
\hline \multicolumn{4}{|c|}{ Receipt of induction chemotherapy } \\
\hline Yes versus no & 2.13 & $1.00-4.53$ & 0.049 \\
\hline \multicolumn{4}{|c|}{ Dysphagia symptoms at diagnosis } \\
\hline Yes versus no & 0.6 & $0.26-1.41$ & 0.243 \\
\hline \multicolumn{4}{|c|}{ Odynophagia symptoms at diagnosis } \\
\hline Yes versus no & 1.51 & $0.55-4.11$ & 0.423 \\
\hline \multicolumn{4}{|l|}{ Weight loss at diagnosis } \\
\hline Continuous variable & 0.95 & $0.90-1.00$ & 0.05 \\
\hline \multicolumn{4}{|l|}{ Total RT dose } \\
\hline Continuous variable & 0.93 & $0.86-1.00$ & 0.041 \\
\hline \multicolumn{4}{|l|}{ PTV volume } \\
\hline Continuous variable & 1 & $1.00-1.00$ & 0.039 \\
\hline \multicolumn{4}{|l|}{ RT modality } \\
\hline IMRT versus 3DCRT & 0.74 & $0.34-1.60$ & 0.44 \\
\hline PBT versus 3DCRT & 0.51 & $0.14-1.91$ & 0.317 \\
\hline \multicolumn{4}{|l|}{ Weight loss during RT } \\
\hline Continuous variable & 0.87 & $0.80-0.95$ & 0.003 \\
\hline \multicolumn{4}{|l|}{ Grade of weight loss } \\
\hline 2-3 versus 0-1 & 0.31 & $0.11-0.84$ & 0.022 \\
\hline \multicolumn{4}{|l|}{ Grade of dysphagia } \\
\hline 2-3 versus $0-1$ & 0.27 & $0.11-0.62$ & 0.002 \\
\hline \multicolumn{4}{|l|}{ Grade of esophagitis } \\
\hline 2-3 versus 0-1 & 0.24 & $0.08-0.69$ & 0.008 \\
\hline
\end{tabular}

Statistically significant $p$-values are in bold.

$\mathrm{Cl}$, confidence interval; AJCC, American Joint Commission on Cancer; RT, radiotherapy; $P T V$, planning target volume; IMRT, intensity-modulated radiotherapy; 3DCRT, three-dimensional conformal radiotherapy; PBT, proton beam therapy.

because early intensive nutritional intervention in patients with upper GI cancers results in significantly improved quality of life and body weight (8). Additionally, intensive nutritional support has been shown to decrease the levels of morbidity associated with chemotherapy and the postoperative period (9).

Our data are consistent with those of head and neck cancers; for instance, investigators determined in a report that nearly half of pFTs were not "used" (defined as $<2$ weeks of feeding) (10). Though the authors did not examine factors that led to "use" of a pFT, our study has not provided justification for pFT insertion in most GE cancers. A notable exception is in cases with tumor obstruction and/or substantial weight loss, which were excluded from our analysis, as these patients often need pFTs-justifiably so-for different circumstances than treatment-related factors alone. This notion may bring forth other issues as to how much weight loss or how much partial obstruction is "substantial" enough to warrant a pFT, in light of other options such as esophageal stenting (11). This may be difficult to test, not only because of the low sample sizes of these patients, but also the large range in clinician/institutional thresholds for pFT insertion.

Limitations to these data are as follows. Though retrospective, there are few prospective data that even detail FT insertion rates; secondary analyses of the many trials examining trimodality therapy are therefore important. The low sample sizes are also noteworthy, but the volume of applicable patients in this study is nearly twice as large as any prospective trial. Our results were not aimed to address factors that are associated with FT or pFT insertion, which would necessitate comparison with the 1,006 patients who did not have FT insertion. Because insertion of (p) FTs is often clinician- and institution-dependent, such results may not be applicable to other institutional policies. Moreover, it is acknowledged that specific reasons for (p)FT placement are also largely institution- and clinician-dependent, limiting applicability to all centers. However, this is a noted limitation facing any study (whether GE cancers or head and neck cancers) examining FTs, and hence may still pertain to practices with somewhat similar reasons for FT placement. Lastly, we excluded 43 patients with post-CRT FT insertion, largely because a proportion undergo placement in anticipation of surgery and not necessarily as a result of toxicities. However, it is still acknowledged that esophagitis peaks 1-2 weeks after CRT, and excluding these patients may miss a proportion of patients with toxicity-related FT placement. This necessitates further research as to which patients are at greatest need for enteral feeding from postoperative complications (12-15).

\section{CONCLUSION}

In GE cancers, with exception of patients having tumor obstruction and/or PWL, these data do not support insertion of FTs prophylactically prior to CRT. In patients receiving induction chemotherapy, higher-dose RT, and/or having lower pretreatment BMI, early and active symptomatic surveillance is indicated in order to intervene before toxicities worsen.

\section{ETHICS STATEMENT}

This study was carried out in accordance with the recommendations of the University of Texas MD Anderson Institutional 
Review Board and Ethics Committee with written informed consent from all subjects. All subjects gave written informed consent in accordance with the Declaration of Helsinki. Permission was obtained to conduct and publish the study.

\section{AUTHOR CONTRIBUTIONS}

VV wrote the manuscript, PA analyzed data, SL supervised the study and conceived of its design. All authors read, edited, and approved the final manuscript.

\section{REFERENCES}

1. Koyfman SA, Adelstein DJ. Enteral feeding tubes in patients undergoing definitive chemoradiation therapy for head-and-neck cancer: a critical review. Int J Radiat Oncol Biol Phys (2012) 84:581-9. doi:10.1016/j.ijrobp.2012.03.053

2. Verma V, Liu J, Eschen L, Danieley J, Spencer C, Lewis JS Jr, et al. Preradiotherapy feeding tube identifies a poor prognostic subset of postoperative p16 positive oropharyngeal carcinoma patients. Radiat Oncol (2015) 10:8. doi:10.1186/s13014-014-0314-3

3. Verma V, Ganti AK. Concurrent chemotherapy in older adults with squamous cell head \& neck cancer: evidence and management. J Geriatr Oncol (2016) 7:145-53. doi:10.1016/j.jgo.2016.01.010

4. Urba SG, Orringer MB, Turrisi A, Iannettoni M, Forastiere A, Strawderman M. Randomized trial of preoperative chemoradiation versus surgery alone in patients with locoregional esophageal carcinoma. JClin Oncol (2001) 19:305-13. doi:10.1200/JCO.2001.19.2.305

5. van Meerten E, Muller K, Tilanus HW, Siersema PD, Eijkenboom WM, van Dekken $H$, et al. Neoadjuvant concurrent chemoradiation with weekly paclitaxel and carboplatin for patients with oesophageal cancer: a phase II study. Br J Cancer (2006) 94:1389-94. doi:10.1038/sj.bjc.6603134

6. Nozoe T, Kimura Y, Ishida M, Saeki H, Korenaga D, Sugimachi K. Correlation of pre-operative nutritional condition with post-operative complications in surgical treatment for oesophageal carcinoma. Eur J Surg Oncol (2002) 28:396-400. doi:10.1053/ejso.2002.1257

7. National Comprehensive Cancer Network. Esophageal and Esophagogastric Junction Cancers. Version 1.2016. (2016). Available from: https://www.nccn. org/professionals/physician_gls/pdf/esophageal.pdf

8. Silvers MA, Savva J, Huggins CE, Truby H, Haines T. Potential benefits of early nutritional intervention in adults with upper gastrointestinal cancer: a pilot randomised trial. Support Care Cancer (2014) 22:3035-44. doi:10.1007/ s00520-014-2311-3

9. Ligthart-Melis GC, Weijs PJ, te Boveldt ND, Buskermolen S, Earthman CP, Verheul HM, et al. Dietician-delivered intensive nutritional support is associated with a decrease in severe postoperative complications after surgery in patients with esophageal cancer. Dis Esophagus (2013) 26:587-93. doi:10.1111/ dote. 12008

10. Madhoun MF, Blankenship MM, Blankenship DM, Krempl GA, Tierney WM. Prophylactic PEG placement in head and neck cancer: how many feeding

\section{ACKNOWLEDGMENTS}

We would like to acknowledge Sameer Rajendra Gajjar, M.D., for his help with data collection for this study.

\section{FUNDING}

Funding was provided in part by The University of Texas MD Anderson Cancer Center and by the National Cancer Institute Cancer Center Support Grant CA016672.

tubes are unused (and unnecessary)? World J Gastroenterol (2011) 17:1004-8. doi:10.3748/wjg.v17.i8.1004

11. Bower MR, Martin RC $2^{\text {nd }}$. Nutritional management during neoadjuvant therapy for esophageal cancer. J Surg Oncol (2009) 100:82-7. doi:10.1002/ jso. 21289

12. Mariette C, De Botton ML, Piessen G. Surgery in esophageal and gastric cancer patients: what is the role for nutrition support in your daily practice? Ann Surg Oncol (2012) 19:2128-34. doi:10.1245/s10434-012-2225-6

13. Lin SH, Wang L, Myles B, Thall PF, Hofstetter WL, Swisher SG, et al. Propensity score-based comparison of long-term outcomes with 3-dimensional conformal radiotherapy vs intensity-modulated radiotherapy for esophageal cancer. Int J Radiat Oncol Biol Phys (2012) 84:1078-85. doi:10.1016/j. ijrobp.2012.02.015

14. Wang J, Wei C, Tucker SL, Myles B, Palmer M, Hofstetter WL, et al. Predictors of postoperative complications after trimodality therapy for esophageal cancer. Int J Radiat Oncol Biol Phys (2013) 86:885-91. doi:10.1016/j.ijrobp.2013. 04.006

15. Juloori A, Tucker SL, Komaki R, Liao Z, Correa AM, Swisher SG, et al. Influence of preoperative radiation field on postoperative leak rates in esophageal cancer patients after trimodality therapy. J Thorac Oncol (2014) 9:534-40. doi:10.1097/JTO.0000000000000100

Disclaimer: This has been presented in part at the 2016 annual meeting of the American Society of Radiation Oncology, September 2016.

Conflict of Interest Statement: SL has research funding from Elekta, STCube Pharmaceuticals, Peregrine, Bayer, and Roche/Genentech, has served as consultant for AstraZeneca, and received honorarium from US Oncology and ProCure. All other authors declare that the research was conducted in the absence of any commercial or financial relationships that could be construed as a potential conflict of interest.

Copyright (C) 2017 Verma, Allen and Lin. This is an open-access article distributed under the terms of the Creative Commons Attribution License (CC BY). The use, distribution or reproduction in other forums is permitted, provided the original author(s) or licensor are credited and that the original publication in this journal is cited, in accordance with accepted academic practice. No use, distribution or reproduction is permitted which does not comply with these terms. 